\title{
Pyoderma gangrenosum associated with erythroid hypoplasia
}

\author{
Cheuk-Kit Wong, Kin-Kee Pun, Christopher C.M. Lum, Shun-Wah Lee, \\ Matthew M.T. Ng and Christina C.L. Wang
}

Department of Medicine, University of Hong Kong, Queen Mary Hospital, Pokfulam Road, Hong Kong.

\begin{abstract}
Summary: Pyoderma gangrenosum is most commonly associated with inflammatory bowel disease and rheumatoid arthritis, but it has been associated with various haematological malignancies. We describe its association with primary erythroid hypoplasia without thymoma in an 80 year old woman who presented with septicaemia complicating urinary tract infection. Spontaneous healing of an extensive lesion was observed.
\end{abstract}

\section{Introduction}

Various myeloproliferative disorders have rarely been found in association with pyoderma gangrenosum. Leukaemia, myeloma, myeloid metaplasia, monoclonal gammopathy and polycythaemia have been so described. ${ }^{1}$ The diagnosis of pyoderma gangrenosum is clinical together with compatible histological findings. ${ }^{2,3}$ We report what is, to our knowledge, the first occurrence of pyoderma gangrenosum in a patient with primary erythroid hypoplasia.

\section{Case report}

An 80 year old woman, known to suffer from primary erythroid hypoplasia for 3 years, was treated with regular blood transfusion. She presented with Escherichia coli septicaemia secondary to urinary tract infection. There was no history of bowel disease, arthritis or peripheral vascular problem. She responded clinically to broad spectrum antibiotic therapy. However, 1 week after admission, multiple erythematous bullous eruptions occurred at the back of her left leg which rapidly ulcerated with ragged undermined margin 2 days later despite local treatment and continuation of antibiotics (Figure 1). The clinical diagnosis was pyoderma gangrenosum. Biopsy of the edge of the ulcer showed a flat ulcer in the skin covered by necrotic tissue and acute inflammatory exudate surrounded by granulation tissue. In the adjacent area, extensive infiltration with polymorph and vascular ectasia was observed with an unremar-

Correspondence: K.K. Pun, M.D., Ph.D., M.R.C.P. Accepted: 8 September 1989 kable epidermis. Cultures of the wound were negative. Blood investigation revealed a haemoglobin level of $10 \mathrm{~g} / \mathrm{dl}$, normal white cell count and platelet count. Rheumatoid factor and anti-nuclear factor were absent. Serum protein concentration and immunoglobulin electrophoresis were normal. A bone marrow biopsy revealed only evidence of depressed erythropoiesis. Computed tomographic scan of the thorax showed no evidence of thymoma. Sigmoidoscopy was normal up to $20 \mathrm{~cm}$. In view of the recent episode of septicaemia, corticosteroid was witheld and she was managed conservatively with local dressing, enteral hyperalimentation and supportive treatment. Complete healing occurred 6 weeks later.

\section{Discussion}

The pathogenesis of pyoderma gangrenosum is unclear. There is no evidence for an infective cause of the lesion. ${ }^{3,4}$ Its association with various autoimmune diseases and its improvement with steroid and the correction of the underlying disorders suggest an immunological basis for the skin lesions. Immunoglobulin deposits and vasculitis consistent with immune-complex disease have been reported. ${ }^{3,5,6}$ Other investigators have suggested alternative theories such as humoral and cellular immunity and dysfunction of neutrophils and monocytes. ${ }^{3,6}$ This diversity of pathogenetic mechanisms reflects our present incomplete understanding of the entity. Indeed, it is not clear whether pyoderma gangrenosum actually represents a distinct pathological entity or whether it is merely the morphologically similar cutaneous manifestation of a variety of disease processes. ${ }^{3}$

In $20 \%$ to $30 \%$ of patients with pyoderma 


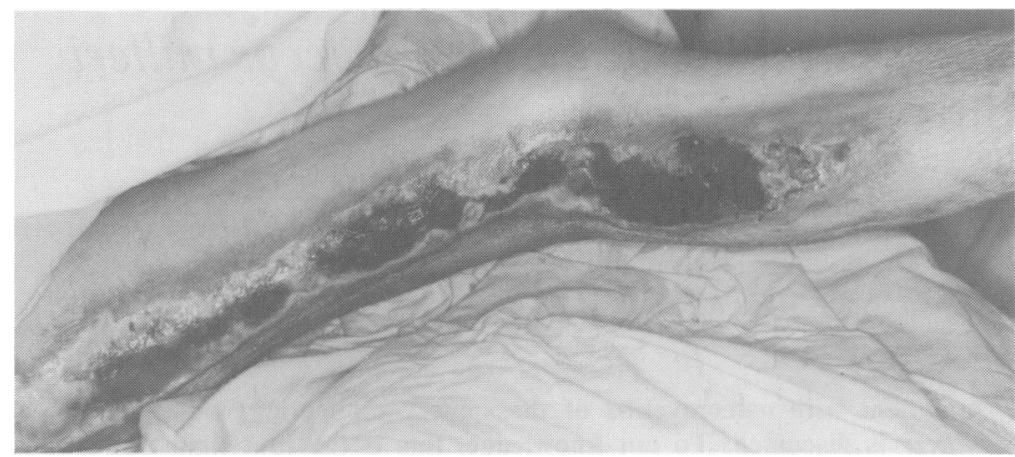

Figure 1 Photograph of the back of the left lower limb showing extensive ulceration with a ragged undermined edge compatible with pyoderma gangrenosum.

gangrenosum, no associated disease process can be identified. ${ }^{3}$ However, in many of these cases, the underlying medical diseases become evident only after a variable period of time. ${ }^{3,7,8}$ The idiopathic cases usually presented to the dermatologist. The lesions tend to be chronic and often persist for years. ${ }^{9}$ In general, treatment is not very satisfactory despite a wide variety of therapeutic agents and relapses often occur. Systemic steroid is the well described treatment although other reported agents include sulpha drugs, clofazimine, disodium cromoglycate, minocycline, cyclophosphamide, benzoyl peroxide, hyperbaric oxygen and immunosuppressive agents. ${ }^{2,3,9,10,12}$ As the cutaneous lesions of pyoderma gangrenosum are often found to parallel the underlying associated disease, such as inflammatory bowel diseases and rheumatoid arthritis, it has been suggested that the beneficial effects of some treatment modalities might be secondary to their effects on the underlying illness. ${ }^{10}$ Indeed, remission of pyoderma gangrenosum can occur after colectomy in patients with ulcerative colitis., ${ }^{3,11}$

In a series of pyoderma gangrenosum complicating haematological malignancy, ${ }^{10}$ a case of healing occurred with induction of complete haematological remission in a patient with acute leukaemia. In the remaining five patients in that series with uncontrolled haematological malignancies, the cutaneous lesions were extensive, failed to heal and led to septicaemia and death of the patients. A few other reports in leukaemic patients have also described healing of pyoderma gangrenosum after successful control of the systemic disease.

Our case appears to be the first case of pyoderma gangrenosum reported complicating erythroid hypoplasia. Despite only supportive treatment, the patient has achieved complete spontaneous healing of the cutaneous lesion. In this instance, the disease course of pyoderma gangrenosum is apparently not related to that of the underlying haematological disease.

\section{References}

1. Haynes, H.A. Cutaneous manifestations of internal malig nancy. In: Braunward, E. (ed.) Harrison's Principles of Internal Medicine. McGraw Hill, New York, 1987, pp. $1588-1593$.

2. Kanel, K.T., Kroboth, F.J. \& Swartz, W.M. Pyoderma gangrenosum with myelofibrosis. Am J Med 1987, 82: $1031-1034$.

3. Schwaegerle, S.M., Bergfeld, W.F., Senitzer, D. \& Tidrick, R.T. Pyoderma gangrenosum: a review. J Am Acad Dermatol 1988, 18: $559-568$.

4. Gilman, A.L., Cohen, B.A., Urbach, A.H. \& Blatt, J. Pyoderma gangrenosum as a manifestation of leukemia in childhood. Pediatrics 1988, 81: 846-848.

5. Schroeter, A. The vasculitis of pyoderma gangrenosum: A dermatopathologic and immunopathologic study, abstracted. Arch Dermatol 1980, 116: 1388.

6. Wolff, K. \& Stingl, G. Pyoderma gangrenosum. In: Fitzpatrick, T.B. (ed.) Dermatology in General Medicine. McGraw Hill, New York, 1987, pp. 1328-1335.

7. Philpott, J.A., Goltz, R.W. \& Park, R.K. Pyoderma gangrenosum, rheumatoid arthritis and diabetes mellitus. Arch Dermatol 1966, 94: 732-738.

8. Hickman, J.G. \& Lazarus, G.S. Pyoderma gangrenosum: a reappraisal of associated systemic diseases. $\mathrm{Br} \mathrm{J}$ Dermatol 1980, 102: 235-237.

9. Ryan, T.J. Diseases of the skin. In: Weatherall, D.J., Ledingham, J.G.G. and Warrel, D.A. (eds) Oxford Textbook of Medicine. Oxford University Press, 1987, 20.67

10. Jacobs, P., Palmer, S. \& Gordon-Smith, E.C. Pyoderma gangrenosum in myelodysplasia and acute leukaemiạ. Postgrad Med J 1985, 61: 689-694.

11. Powell, F.C., Schroeter, A.L., Su, W.P. \& Perry; H.O. Pyoderma gangrenosum: a review of 86 patients. $Q J$ Med 1985, 55: 173-186.

12. Stanley, J.L., Poh-Fitzpatrick, M.B. \& Walther, R.R. Atypical pyoderma gangrenosum with leukemia. JAMA 1978, 239: 935-938. 